\title{
$\operatorname{con} 6.790415 \cdots 22$
}

The "Cleanex" Process: A Versati.le Solvent Extraction Process for Recovery and Purification of Lanthanides, Americium, and Curium*

John E. Bigelow, Emory D. Collins, and Lester J. King

Oak Ridge National Laboratory

P. O. Box $X$

Oak Ridge, Tennessee 37830

To be presented at the American Chemical Society/Chemical Society of Japan Chemical Congress, Honolulu, Hawaii, April 1-6, 1979

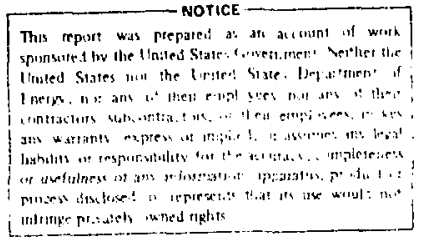

BV acceptance of this article, the publisher or recipient acknowledges the U.S. Government's right to retain a nunexclusive, rovalty. frea licenso in anel to any copyright covering the article.

*

Research sponsored by Office of Basic Energy Sciences, U.S. Dopartment of Energy, undor contract W-7405-eng-26 with the Union Carbide Corporation.

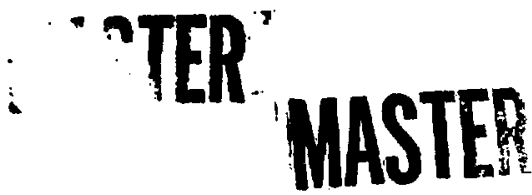


The "Cleanex" Process: A Versatile Solvent Extraction Process for Recovery and Purification of Lanthanides, Americium, and Curium*

John E. Bigelow, Emory D. Collins, and Lester J. King

Oak Ridge National Laboratory

P. O. Box $\mathrm{X}$

Oak Ridge, Tennessee 37830

\begin{abstract}
The "Cleanex" solvent extraction process makes use of the extractive reagent di(2-ethylhexyl) phosphoric acid (IDEit?). nt a concentration of $1 \underline{M}$ in straight-chain hydrocarbon diluent, HDEHP will extract americium, curium, and other trivalent actinide and lanthanide elements from dilute acid or salt solutions. The solute is back-extracted with more concentrated acid, either nitric or hydrochloric. The process has been used in the continuous, countercurrent mode, but its greatest advantage arises in batch extractions where the excess acid can be titrated with $\mathrm{NaOH}$ to produce a final acidity of about $0.03 \mathrm{M}$. Under these conditions, 99\% recovery can be achieved, usually in one stage. We have used cleanex on the 50liter scale at the Transuranium Processing Plant at Oak Ridge for 12 years to provide a broad spectrum cleanup to transuranium elements before applying more sophisticated techniques for separating individual products. The process is also used routinely to recovor excessive losses of curium and/or californium from plant waste streams. The solvent system is relatively resistant to radiation damage, being usable ip to $200 \mathrm{w}$-hr/liter.
\end{abstract}

* Research sponsored by Office of Basic Energy Sciences, U.S. Department of Energy, under contract W-7405-eng-26 with the Union Carbide Corporation. 
The "Cleanex" solvent extraction process features use of the extractive reagent, di(2-ethylhexyl) phosphoric acid (HDEHP), a liquid cation exchanger, to extract the trivalent actinide and lanthanide elements. The name "Cleanex" was coined because the process was initially developed to "clean up" several batches of rework solutions from a wide variety of contaminants. The process worked so well that it was latex incorporated into the mainline operations at the Transuranium Processing Plant (TRU) at tile Uar Ridige National Laboratory (ORiv), where it has been in routine uae for 12 years. At TRU, the process has been applied to a number of different baich solvent extraction operations at the 50-liter scale. These include purification of solutions of transplutonium elements, conversion of the solut.ion anion from nitrate to chloride, and recovery of transplutonium elements from rework and waste streams. ${ }^{1}$ The "Cleanex" process was adapted from the Dapex process, ${ }^{2}$ which has been used for many years in the uraniun milling industry.

The chemistry, reagents, equipment, basic operations, and typical results obtained with the "Cleanex" process are covered in the succeeding sections.

\section{PROCESS CHEMISTRY}

The process consists of liquid-liquid solvent extraction of trivalent actinides (transplutonium elements) from a dilute acid or salt solution in which the anion can be either nitrate or chloride. The relatively inextractable impurities can be scrubbed from the pregnant organic phase with dilute acid $(0.03 \underline{M})$ and the solutes back-extracted, or stripped, with more 
concentrated acid (2-6 M). The process works with nitric or hydrochloric acid or mixtures of the acids. Since the extracted species does not contain complexed acid, the solutes may be stripped into a different acid than the one from which they were initially extracted. Thus, the process may be used to convert a nitrate solution to a chloride solution.

The organic extractant is the ester, bis-2-ethylhexyl hydrogen phosphate, which has acidic properties because of the replaceable hydrogen ion, and is usually called in the trade di(2-ethylhexyl) phosphoric acid or HDEHP. This reagent has a relatively high density and viscosity so it is usually diluted with a nor-polar solvent to a concentration of about 1 molar to produce an organic extractant phase with suitable operating characteristics. In the cleanex process, aliphatic diluents are preferred over aromatic diluents because of about a 5-fold higher extraction coefficient for the trivalent actinides.

The HDEHP is a cation exchanger and its extraction of trivalent metal ions $\left(M^{+3}\right)$ can be expressed by the following chemical reaction:

$$
\mathrm{M}_{\mathrm{aq}}^{+3}+3(\mathrm{HDEHP})_{\text {org }}+\mathrm{M}(\mathrm{DEHP})_{3, \mathrm{Org}}+3 \mathrm{H}_{\mathrm{aq}}^{+}
$$

At equilibrium, the concentrations of the reacting species are related as follows (neglecting activity coefficients): :

$$
K=\frac{\left[\mathrm{M}(\mathrm{DEHP}) 3^{3} \text { org }\left[\mathrm{H}^{+}\right]_{\mathrm{aq}}^{3}\right.}{[\mathrm{HDEHP}]_{\text {org }}^{3}\left[\mathrm{M}^{+3}\right]_{\mathrm{aq}}},
$$

where $\mathrm{K}$ is the equilibrium constant for the reaction. The equilibrium extraction coefficient $(E)$ for the trivalent metal, defined as the ratio of the concentration in the organic phase to the concentration in the aqueous phase at equilibriurn, can be obtained by a rearrangement of Eq. (2): 


$$
E=\frac{\left[M(D E H P)_{3}\right]_{\text {org }}}{\left[\mathrm{M}^{+3}\right]_{\mathrm{aq}}}=\frac{K[\mathrm{HDEHP}]^{3} \text { org }}{\left[\mathrm{H}^{+}\right]_{\mathrm{aq}}^{3}} .
$$

Thus, the extraction coefficient, E, varies inversely with the third power of the aqueous phase acidity $\left(\mathrm{H}^{+}{ }_{\mathrm{aq}}\right)$, and directly with the third power of the uncombined HDEHP.

The acid dependency observed in practice has been only approximately inverse third power. Impurities in cleanex feed solutions often cause a departure from ideality (e.g., by common-ion effect, or by consumption of some of the uncombined HDEHP), and we have not been able to control the extraction of the actinide elements solely by monitoring the aqueous phase acidity.

\section{A. Materials}

(1) Feed Solutions - The process is applicable to solutions of trivalent actinides or lanthanides in either dilute acid or a variety of salt solutions in either nitrate or chloride or mixed media. Sodium concentrations up to 3 M can be tolerated, but lithium begins to have adverse effects above $2 \underline{M}$ and potassium is objectionable even at $0.5 \underline{\text { s. Certain }}$ special constituents can be tolcrated in the feed if specific precautions (described later) are taken.

(2) Extractive reagent - The extractive reagent is commercial grade di(2-ethylhexyl) phosphoric acid (HDEHP). Various lots of material obtained from Mobil, from Stauffer, and from union Carbide, have all appeared equally useable. The commercial material typically contains 1-1.5\% of the monoester (which is diacidic) and a small amount of inert material, probably unreacted 2-ethylhexanol and pyrophosphoric acids. The HDEHP generally titrates about $98 \%(2.8 \mathrm{M})$ and is used without treatment. 
(3) Diluents - Almost any aliphatic diluent will do but a high flash point is preferred for safety reasons. At TRU, Amsco 125-82 has been used in the past, and then NFH (normal paraffin hydrocarbon) supplied by The South Hampton Co., Silsbee, Texas. Our current diluent is odorless mineral spirits supplied by Amsco, and having a flashpoint of $52^{\circ} \mathrm{C}$, a specific gravity of 0.750 , and a boiling range of $144-208^{\circ} \mathrm{C}$. All of these diluents have been satisfactory, and no effort has been made to systematically evaluate the different diluents for our applications. We generally pretreat the diluent by passing it through a packed column of silica gel and then alumina to remove traces of surface-active agents that might later contribute to the formation of scable emulsions.

\section{B. Equipment}

The equipment typically used at TRU for carrying out the cleanex process batchwise is illustrated schematically in Fig. 1. The operating technique and Jesign ccinsiderations for some or the items in Fig. 1 are more fully described by Chattin. ${ }^{3}$ The feed is adjusted and the extraction is carri=d out batchwise in a tantalum-lined evaporator with a capacity of 60-70 1 iters. Phase contact is accomplished by air-sparging. A vacuum transfer system is used to raise the aqueous phase from the bottom of the tantalum evaporator, through a phase separator (or bull's-eye) and into a vacuum transfer tank. Replacing the vacuum source with a low pressure air line, and operating a valve, permits discharge of the accumulated aqueous phase from the vacuum transfer tank into either a waste receiver or product 
receiver tank. The raffinates (including scrubs) eventually are transferred to a waste storage tank. The product receiver is usually another tantalumlined evaporator so that successive volumes of strip acid can be combined and concentrated prior to their introduction into the next step in the overall process.

\section{Processing Steps}

(1) Feed Adjustment - The product from a previous step is transferred and flushed into the tantalum-lined evaporator. Most of the excess acid contained in the composited cleanex feed is removed by evaporation either to a final volume of 2 liters or, if the salt content is high, to a final boiling temperature of $130^{\circ} \mathrm{C}$, whichever occurs first. If the feed is a nitrate solution, hydrolysis of some elements, e.g., zirconium, may have occurred during the evaporation; therefore, about 1 liter of $12 \mathrm{M} \mathrm{HCl}$ is added and the feed solution is reheated to $120^{\circ} \mathrm{C}$ to redissolve any hydrolyzed materials. The feed is then diluted with water to make a total volume of 12 liters and is mixed and sampled. An addition of acid or NaOH is made (if necessary) to adjust the solution acidity into the range of 0.1 to $0.4 \mathrm{~N}$. An oxidant solution ( $1.5 \mathrm{MLiOCl}$ ) is added to make the feed $0.1 \mathrm{M}$ LiOCl, and the feed solution is then heated to $80^{\circ} \mathrm{C}$ and digested for 20 min to remove any gaseous reaction products. The cxidation and digestion is carried out to ensure that all molybdenum in the feed is oxidizcd to the extractable Mo(VI) form. ${ }^{4}$ otherwise, the unextracted molybdenum may hydrolyze in the relatively low-acid conditions achieved later in the extraction step and result in severe emulsion problems.

(2) Extraction - About 30 liters of the previously prepared organic extractant is added. Adequate mixing of the phases is achieved by air sparging at a superficial air velocity of $0.25 \mathrm{ft} / \mathrm{min}$ (this is the same velocity used to mix a single phase prior to sampling). The total phase-mixing (sparging) time is typically 3 to 4 hours. 
After mixing has begun, an amount of $\mathrm{NaOH}$ calculated to reduce the aqueous phase acidity to $0.03 \mathrm{~N}$ is added. (This lower limit of acidity has been chosen to minimize the possibility of hydrolysis and precipitation of unextracted feed components, which might lead to emulsification.) The phases are mixed 30 to $60 \mathrm{~min}$ and chen allowed to settle. The aqueous phase is sampled and analyzed for free acid and for gross alpha (primarily ${ }^{244} \mathrm{Cm}$ ). The free acid is almost always higher than $0.03 \mathrm{~N}$ because acid is transferred to the aqueous phase in exchange for the metallic cations which were extracted into the organic phase.

A new NaOk addition is calculated, based on the acid results, and the acid adjustment and sparging are repeated until the amount of unextracted curium remaining in the aqueous phase is less than the specificd limits or if successive analyses show the amount of unextracted curium has begun to increase. (This latter phenomenon is believed due to displacement of the curium by aluminum resulting from a slow, irreversible extraction of aluminum which consumes the reagent, HDEHP.)

At this point, the aqueous raffinate is transferred to a separate tank and the pregnant organic phase is subsequently scrubbed and stripped in the same vessei.

(3) Scrubbing - Since a relativaly large amount of sodium (as NaOH) is added during a clcanex extraction to neutralize excess acidity, sodium is usually the predominant metallic impurity. A very small portion of the sodium is extracted by the HDEHP; this amount can be reduced by scrubbing the pregnant organic with $0.03 \mathrm{~N}$ acid. A second function of the scrubs, and probably the most important function, is to flush the residual, unextracted impurities from the aqueous heel underlying the pregnant organic layer. 
The pregnant organic phase is usually scrubbed twice with $0.03 \mathrm{~N} \mathrm{HCl}$ (the lst scrub is $0.03 \mathrm{~N}^{\mathrm{NNO}} 3$ j.f the feed solution had been in the nitrate form). The volume of each scrub is $1 / 3$ of the organic phase volume. Each scrub is mixed with the organic phase by air-sparging at least 30 minutes. The scrub raffinates are usually combined with the extraction raffinate to provide a composite feed for a secona-stage extraction.

(4) Organic Phase Modification and Stripping - Iron is frequently a significant impurity in cleanex feels and, if present, it will have been oxidized to Fe(III) by radiolysis and by the Jiocl which was added to the feed. The Fe(III) would tend to follow the transplutonium elements through the extraction and stripping steps. In order to retain the Fe(III) in the organic phase while the transplutonium elements are stripped, a solution of 1.6 M Aclogen 364-HP (a straight-chain tertiary amine) in diethylbenzene (DEB) is added to the pregnant cleanex organic phase just before stripping. The amount of Adogen added is enough to make the organic phase $0.2 \mathrm{M}$ anine. Following this modification of the orginic phase, the transplutonium elements are stripped with $6 \mathrm{MHCl}-0.5 \mathrm{M} \mathrm{H}_{2} \mathrm{O}_{2}$. The hydrogen peroxide in this solution serves to ensure that all borkelium is reduced to the trivalent state so that it can be stripped. However, the oxidation-reduction potential is not sufficient to rcduce and strip any Pu(IV) impurity that might be present. $\mathrm{t}:$ is always necessary to strip the organic phase with several batches of strip solution in order to get a.ll of the transplutonium elements transferred to the collection tank. Stripping coefficients for the transcurium elements are lower than for curium; thus, the amounts of californium remaining in the organic phase and in the aqueous heel solution (as indicated by gross neutron counts) are monitored to confirm the completeness of the stripping operations. 
(5) Exposure of the Organic Extractant to Radiution - Fadiolytic damage to the organic extractant is quite likely to occur since ${ }^{244} \mathrm{Cm}$ (2.83 watts $/ g$ ) is present in the organic phase foi a significant time. Two hundred watt-hours/liter $\left(8 \times 10^{7} \mathrm{rad}\right)$ is taken to be the upper limit of allowable exposure and at TRU we generally try to hold planned exposure below 150 watt-hours/liter. Thus, in the case of a feed containing enough ${ }^{244} \mathrm{Cm}$ to generate 5 watts/liter (in the organic phase), the extraction and scrubbing must be completed, and stripping begun within 30 hours. We have not experienced any operating difficulties with Cleanex runs made under these conditions, but: several samples of curium oxide product have been found that contained 5 to $8 \%$ phosphorus, presumably from radiolytic decomposition of the HDEHP. Potentially, the process of decomposition can form extractant species that behave quite differently from HDEHP and cause a variety of deleterious effects. Increasing difficulty in stripping has been observed on occasions when the exposure approached 200 watt-hours/liter.

(6) Oxganic Phase Entrainment During Nqueous Pisase Transfers - One advantage of batch solvent extraction processes is that adequate time can be allowed for the organic and aqueous phases to settle and separate. The aqueous (bottom) phase can then be transferred to a separate tank using a special phase-separator vessel. Although the phase-separator is very effective in preventing bulk transfer of the wrong phase, a small amount of the organic phase is frequently entrained in the aqueous phase. If the next processing step requires evaporation of the Cleanex product solution to a small volume, the entrained HDEHP could be decomposed to a tar which could sorb a significant amo nt of the actinide elements. When this possibility exists, the transfer of cleanex product solution to the evaporator is made via a tank containing a few liters of organic diluent (DEB or imsco) so that a second, cleaner phase separation can be made. Possible entrainment 
of the pure diluent into the product is of little consequence since the diluent will steam-distill out of the receiving tank during evaporation! of the aqueous product.

\section{RESULTS}

Typical results of the cleanex process, as applied to a HFIR target processing campaign, are shown in Table I. Part A shows the quantities of the transplutonium elements found in a typical target campaign, and their behavior in tho process. Part $B$ shows the behavior of major fission products, and part $C$ shows the purification that can be obtained from common macroscopi.c contaminant ions.

\section{CONCLUSTONS}

Tho cleanex process is particularly well suitel to be carried out as a batch process. The extraction coefficients are strong functions of the aqueous phase acidity; thus, for complete extraction it is important to reduce the acidity to the minimum practical value. Howevir, as the acidity is reduced, more of the solute ions extract into the organic phase, where they undergo a cation exchange reaction with the extraction reagent (HDEHP), thus releasing free acid. Finis fxee acid rill back-extract into the aciueous phase, where it will strongly affect the extraction coeffiriant. A step-wise addition of NaOH allows one to "titrate" the free acid, achieving almost any desired degree of extraction. Furthermore, the precision of acid control that this affords allows one to control the reaction pathway so that certain extractable but easily hydrolyzed cations, such as $\mathrm{Zr}(\mathrm{IV})$, can be completely removed from the aqueous phase before the acid concentration is decreased to its final value. This 
significantly reduces the potential of these impurity ions to form interfacial cruds. Even if some crud is formed, and phase separation is thereby delayed, a batch extraction does not require a rapid phase separation, and thus is tolerant of a very dirty solution.

In the environment of the TRU Facility at ORNL, the cleanex process has proven to be extremely valuable, and is rolitinely relied upon to perform a varjety of process tasks. 


\section{Literature Cited}

1. Collins, E. D., Bigelow, J. F., Proc. 24th Conf. Remote Syst. Technol., 1976, pp, 130-139.

2. Brown, K. B., Coleman, C. F., Crouse, D. J., Blake, C. A., and Ryon, A. D., "Solvent Extraction Processing of Uranium and Thorium Ores," 2nd Int' 1. Conf. on the Peaccful Uses of Atomic Energy, $A / C o n f .15 / P / 509$, sept. 1958.

3. Chattin, F. R., King, L. J., Peishel, F. I., Proc. 24th Conf. Remote Syst. Technol., 1976, pp. 118-129.

4. Weaver, B. S., private Communication, May 1957. 
Table I. Typical Composition of Cleanex Feed Solutions when Processing Transplutonium Elements During a HFIR Target Campaign

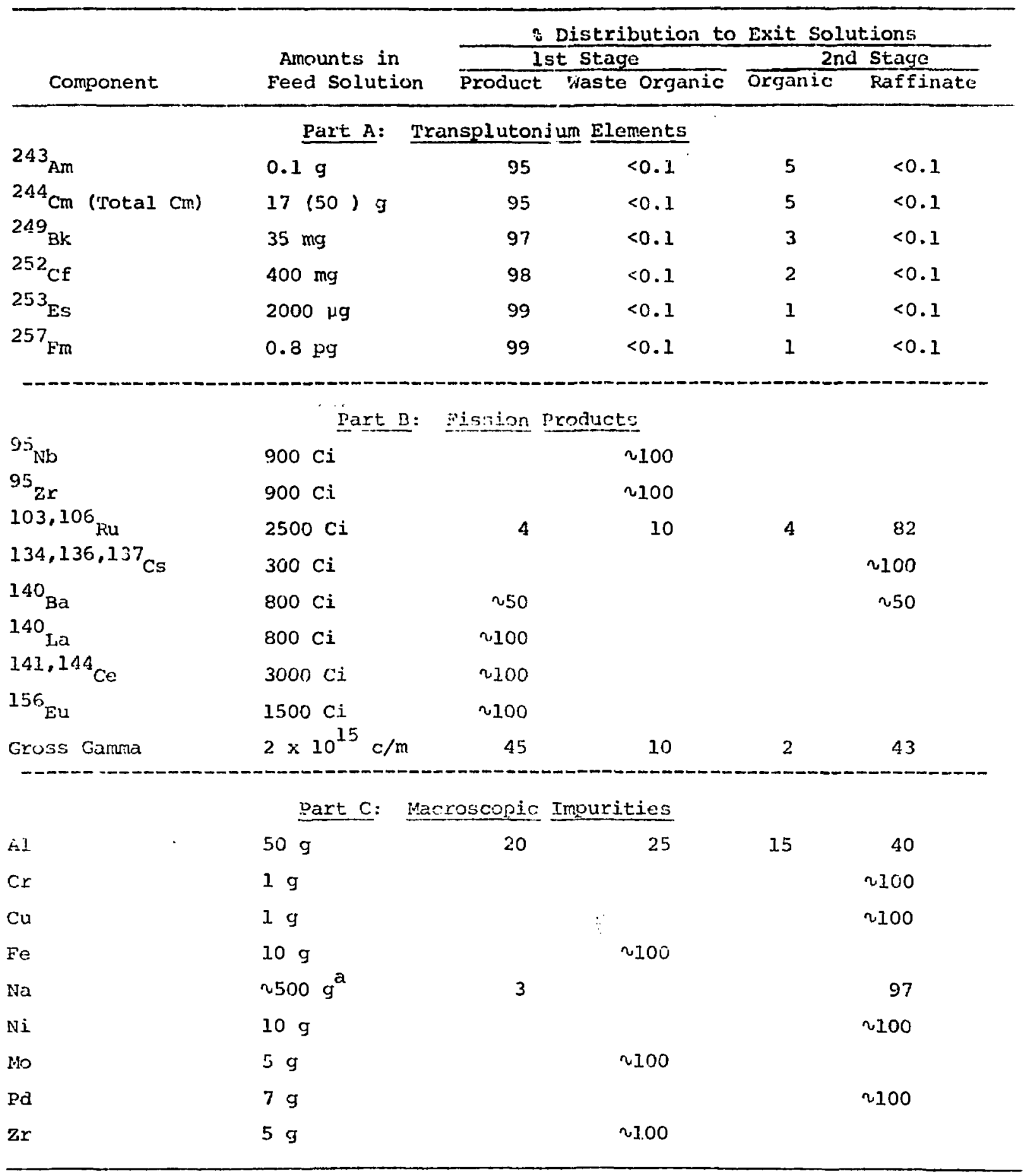

a Several hundreds of grams of $\mathrm{Na}$, as $\mathrm{NaOH}$, are added to neutralize the aqueous phase acidity. 


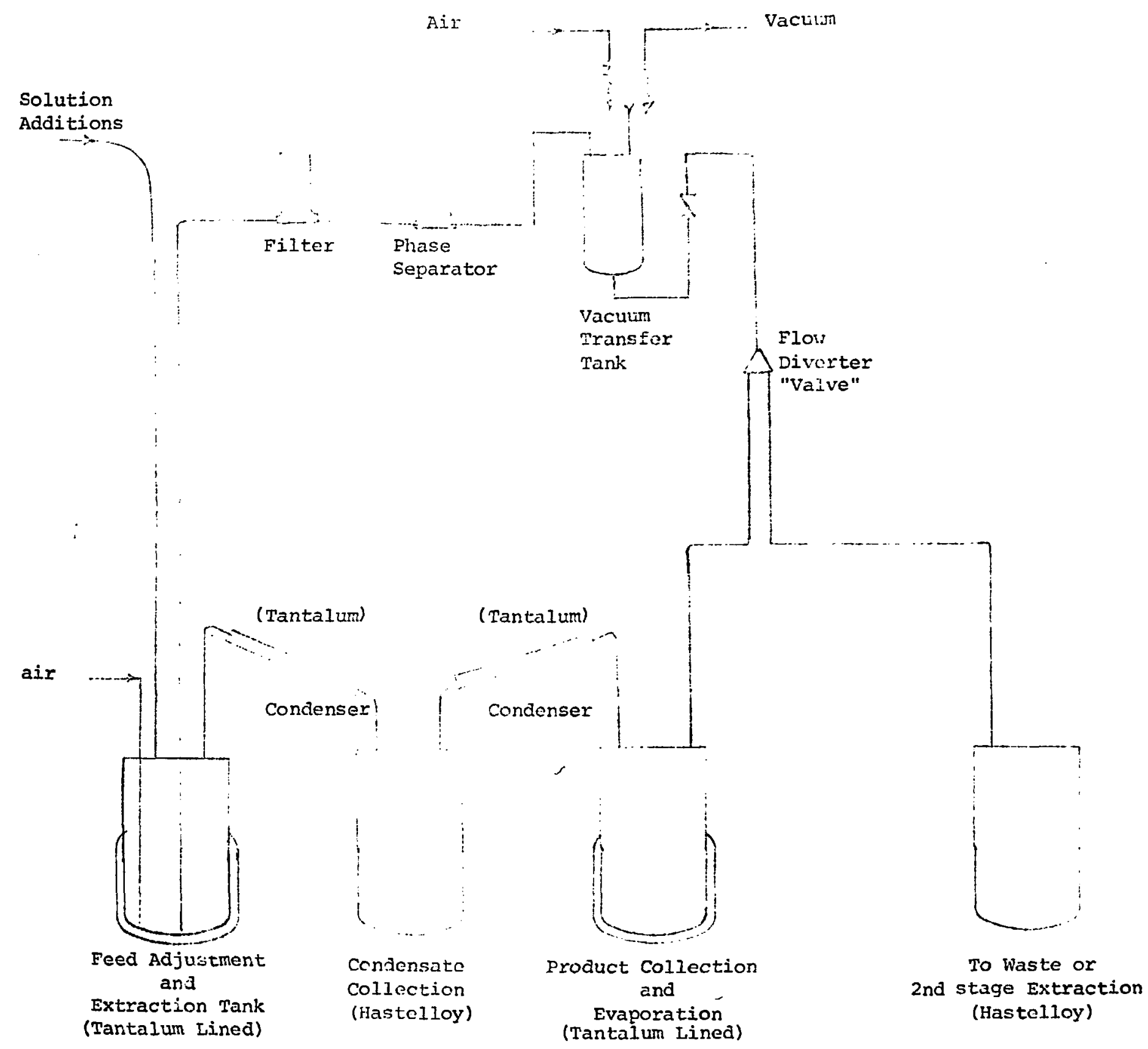

